\title{
Comparison of perceptual and motor latencies via anticipatory and reactive response times
}

\author{
Pedro Cardoso-Leite, Pascal Mamassian, and Andrei Gorea \\ Université Paris Descartes and CNRS, Paris, France
}

\begin{abstract}
To compare the timing of perceptual and motor decisions, distinct tasks have been designed, all of which have yielded systematic differences between these two moments. These observations have been taken as evidence of a sensorimotor dissociation. Inasmuch as the distinction between perceptual and motor decision moments is conceptually warranted, this conclusion remains debatable, since the observed differences may reflect the dissimilarity between the stimulations/tasks used to assess them. Here, we minimize such dissimilarities by comparing response time (RT) and anticipatory RT (ART), an alternative technique with which to infer the relative perceptual decision moments. Observers pressed a key either in synchrony with the third of a sequence of three stimuli appearing at a constant pace (ART) or in response to the onset of this third stimulus presented at a random interval after the second (RT). Hence, the two stimulation sequences were virtually identical. Both the mean and the variance of RT were affected by stimulus intensity about 1.5 times more than were the mean and the variance of ART. Within the framework of two simple integration-to-bound models, these findings are compatible with the hypothesis that perceptual and motor decisions operate on the same internal signal but are based on distinct criteria, with the perceptual criterion lower than the motor one.
\end{abstract}

Understanding how visual stimuli give rise to visual and to motor responses has been a main issue in cognitive neuroscience since its foundation (for reviews, see Glimcher, 2003; Jeannerod, 1997; Milner \& Goodale, 1995). One psychophysical approach to the investigation of the sensorymotor relationship consists in confronting perceptual and motor latencies (e.g., Jaśkowski, 1996, 1999; Jeannerod, 1997). The rationale of this slant is that an independent processing of information for action and for perception should yield differences in the temporal layout of the perceptual and motor responses, whereas a common sensory-motor processing system should not. This approach, together with its different empirical realizations (including the present one), does not prejudge the definition of the very concepts of perceptual and motor decision moments nor their relative timing, although it links motor decisions to low-level online reactive processes and perceptual decisions to higher level processes less dependent on the immediate sensory input. What it does prejudge, however, is the very existence of these two types of moments and, therefore, the possibility of their independent measurement. Denying this possibility amounts to rendering the whole sensorimotor dissociation debate groundless.

In this study, we go along with the standard contemporary terminology whereby the "nature" of a percept is defined by the very task used to assess it - namely, detection (as in the present experiments), discrimination, identification, or classification. ${ }^{1}$ In the remainder of this article, the timing of such processes will be referred to as the moments when the relevant internal activity exceeds a decision criterion, as classically defined by signal detection theory (Green \& Swets, 1966). Accordingly, any difference between perceptual and motor decision moments should be attributed to distinct perceptual and motor criteria, to different time constants of the respective internal responses (the strong sensorimotor dissociation stand), or to both.

The most widespread method used to compare perceptual and motor latencies (for a review, see Jaśkowski, 1996) consists in pitting the difference in simple response times $(\Delta \mathrm{RT})$ to stimuli of unequal saliency against the point of subjective simultaneity (PSS) for these same stimuli as assessed from temporal order judgments (TOJs). This comparison is based on the largely consensual notion that the RT task is a direct way of assessing motor latencies, whereas the TOJ task, in order to be performed, requires the comparison of two perceptual moments.

The simplest model of the sensory-motor relationship posits that RT and TOJ tasks rely on the same internal signal and on the same decision (Gibbon \& Rutschmann, 1969). Accordingly, such a model predicts that, in response to two unequally salient stimuli, motor $(\Delta \mathrm{RT})$ and perceptual (PSS) delays should coincide. Most experiments, however, have shown that PSS and $\triangle \mathrm{RT}$ (and, more generally, perceptual and motor latencies) are differently affected by a number of stimulus manipulations (for reviews, see Jaśkowski, 1996, 1999; Miller \& Schwarz, 2006). In particular, the effect of manipulations of stimulus intensity on $\Delta \mathrm{RT}$ has been shown to be about double 
the effect on PSS (Jaśkowski, 1992; Jaśkowski \& Verleger, 2000; Roufs, 1974; but see Roufs, 1963).

In addition to neuropsychological reports (Goodale \& Milner, 1992) and to a number of psychophysical masking studies (e.g., Klotz \& Neumann, 1999; Schmidt \& Vorberg, 2006; Vorberg, Mattler, Heinecke, Schmidt, \& Schwarzbach, 2003), the $\Delta$ RT-PSS discrepancy has been taken as yet another piece of evidence in favor of two independent motor and perceptual processing systems (Neumann, Esselmann, \& Klotz, 1993; Tappe, Niepel, \& Neumann, 1994). This dissociation, nonetheless, has been firmly criticized on anatomo-physiological (e.g., Merigan \& Maunsell, 1993), behavioral (e.g., Franz, Gegenfurtner, Bülthoff, \& Fahle, 2000; Gegenfurtner \& Franz, 2007; Stone \& Krauzlis, 2003), and even neuropsychological (e.g., Pisella, Binkofski, Lasek, Toni, \& Rossetti, 2006; Rossetti, Pisella, \& Vighetto, 2003; Schenk, 2006) grounds.

An intermediate path proposes that perceptual and motor decisions operate either on the same internal response, but at different times and internal activation levels, or according to different decision rules. Sternberg and Knoll (1973), for example, proposed that motor responses are triggered as the internal activation evoked by the stimuli exceeds a motor threshold, whereas the perceptual judgments are based on the temporal delay between the peak values of these internal evoked responses, a concept with no biological foundation (Lennie, 1981). More recently, Miller and Schwarz (2006) presented a one-system diffusion model in which TOJ and RT are triggered as a unique diffusion signal reaches first the perceptual and then the motor thresholds (or bounds), respectively. Such a one-signal-two-decisions integrationto-bound view had already been proposed by Sanford (1974) and has been backed up by a number of behavioral studies (e.g., Cardoso-Leite, Gorea, \& Mamassian, 2007; Ejima \& Ohtani, 1987; Waszak, Cardoso-Leite, \& Gorea, 2007; Waszak \& Gorea, 2004). For example, the systematically observed correlation between RT and TOJ, with $\Delta \mathrm{RT}$ larger than PSS by a constant ratio, can easily be accounted for in terms of such one-signal-two-decisions models (see Cardoso-Leite et al., 2007; Miller \& Schwarz, 2006).

Despite this consistently reported $\triangle \mathrm{RT}-\mathrm{PSS}$ difference, the specific relationship between $\Delta$ RT and PSS may drastically vary within and across studies (e.g., Aschersleben \& Müsseler, 1999; Cardoso-Leite et al., 2007; Jaśkowski, 1992, 1993; Roufs, 1974; Tappe et al., 1994). Standard one-system integration-to-bound models require that the variances of the perceptual and motor responses decrease in proportion to stimulus intensity and increase with the buildup of these responses over time. It follows that the relationship between mean perceptual and motor decision times, on the one hand, and the relationship between their variances, on the other hand, should follow a function critically dependent on the underlying decision processes. Cardoso-Leite et al.'s (2007) study is the only one we know of that has empirically addressed this issue. That study showed that although perceptual and motor decision times (as derived from RT and TOJ measurements) are linearly related with regard to both their means and their standard deviations, the respective slopes differ significantly. In particular, whereas the stimulus intensity effects on the TOJ means were about 2.1 times larger than those on the RT means, these same effects on the TOJ standard deviations were about 1.5 times smaller than those on the RT standard deviations. On the basis of their intuition that a simplified one-system-two-decisions integrationto-bound model should predict equal means and standard deviation slopes, ${ }^{2}$ these authors suggested that the larger TOJ scatter is inherent to this experimental paradigm.

RT and TOJ measurements differ in a number of ways. The two temporally close stimuli used in TOJ may yield apparent motion (a cue that might be useful for the TOJ task, but not for the RT task) or may mask each other unequally (with the occasional transient fading of the lower saliency stimulus; Kanai \& Kamitani, 2003). The inherent subjective nature of the TOJ task permits the use of a range of temporal markers (e.g., Jaśkowski, 1992; Sternberg \& Knoll, 1973) and is prone to uncontrolled contextual effects (e.g., Miyazaki, Yamamoto, Uchida, \& Kitazawa, 2006). These factors may generate a significant increase in the variability of the data. Finally, TOJs are not time constrained and, therefore, allow the use of information that is not accessible in the speeded RT task. For all these reasons, the comparison of RT and TOJ performances remains a subject of debate.

The purpose of the present study is twofold. First, it introduces a method of assessing perceptual latencies presumably less prone to the criticisms above. Second, it develops two quantitative models to predict the relationship between perceptual and motor decision latencies with regard to both their means and their variances.

\section{The Anticipation Response Time Method and Its Rationale}

The measurement of RTs to periodic stimuli has been used at least since Stevens (1886) to study time perception. It has been declined in at least two forms. The most frequently used form consists of having subjects synchronize their tapping with a series of equally time-spaced (auditory or visual) stimuli. Alternatively, subjects are exposed to only three equally time-spaced events and are asked to press a button in synchrony with the third event (e.g., Doehring, 1961; Mamassian, 2006, 2008). We will refer hereafter to the latter procedure as the anticipation RT (ART). Note that since this anticipatory response is a delayed response to the second stimulus in the sequence, ART is, according to the implicit definition commonly used in the literature, a measure of perceptual latency. The latency derived from tapping is, however, less appropriate than ART for deriving a perceptual latency. In tapping, subjects typically enter an automatic response mode so that their response pace becomes partly self-sustained and, hence, less dependent on the periodic stimulus with which it must be synchronized. Also, inasmuch as the latency to be derived from tapping is to be compared with the motor latency assessed via RT, the two tasks cannot be matched, since, by construction, RT cannot be automatized. Finally, although not critically, tapping typically entails a negative asynchrony error (e.g., Aschersleben, 2002), whereas ART does not (Mamassian, 2006, 2008). ${ }^{3}$ Strangely, the reproduction of time intervals for the purpose of testing the sensorimotor dissociation 
has been rarely used and only with the tapping procedure (Aschersleben, 2002; Aschersleben \& Müsseler, 1999). This study is the first to use ARTs for assessing perceptual latencies and to compare them with RTs.

Figure 1 illustrates the rationale underlying the present ART technique. Three identical stimuli are presented in sequence at a constant pace. They can be of relatively low (black bars) or high (gray bars) saliency (Figure 1A). The standard assumption is that the buildup over time of the internal responses evoked by each stimulus in the sequence-equivalent to the accumulation of evidence in favor of stimulus presence-is proportional to the stimulus's saliency (Carpenter \& Williams, 1995; Gold \& Shadlen, 2001; Luce, 1986). The internal responses to low-saliency stimuli (black oblique lines) will reach a perceptual decision criterion (dashed horizontal black line labeled $c_{\mathrm{P}}$ in Figure 1B) after the internal responses to the higher saliency stimulus (gray oblique lines). Hence, the ART for the low-saliency stimulus will be delayed by as much, with respect to the ART for the higher saliency stimulus, with the ART difference ( $\triangle \mathrm{ART}$ ) proportional to the difference in stimulus saliency. ${ }^{4}$ This reasoning holds whether the accumulation of evidence is described by a linear deterministic (as in Figures 1B and 1C; see, e.g., Brown \& Heathcote, 2008; Grice, 1972; Reddi, Asrress, \& Carpenter, 2003; Reeves, Santhi, \& DeCaro, 2005) or stochastic (e.g., Luce, 1986; Wald, 1947) function.

For generality, we will assume, along with a number of authors (see above), that perceptual and motor behaviors are triggered at distinct response levels - that is, for different perceptual, $c_{\mathrm{P}}$, and motor, $c_{\mathrm{M}}$, criteria (Figure 1C) - but that they are based on the same internal response. The TOJRT literature indicates that RTs are affected by saliency changes more than TOJs, hence suggesting that $c_{\mathrm{P}}<c_{\mathrm{M}}$. On the basis of experiments using a different task and methodology, the $c_{\mathrm{P}}>c_{\mathrm{M}}$ case has also been advanced (Waszak et al., 2007), suggesting that the relationship between motor and perceptual criteria is stimulus/task dependent.

\section{Modeling the RT-ART Relationship}

We present two integration-to-bound decision models and their predictions of the RT-ART relationship; both models yield a linear relationship between ART and RT means, on the one hand, and between their associated variances, on the other hand. ${ }^{5}$ The two models predict that the slope of the relationship between ART and RT means is given by the $c_{\mathrm{P}} / c_{\mathrm{M}}$ ratio, but they predict different slopes for the relationship between ART and RT variances.

The measured response time (RT or ART) distributions for a given stimulus intensity, $i, \tau(i)$, are considered under both models presented below as the sum of two random variables,

$$
\tau(i)=\delta(i)+k,
$$

with $\delta(i)$, a random variable representing the moment of detection of a stimulus of intensity $i$, and with $k$, a combined task + observer specific random variable independent of the decision process. ${ }^{6}$ The mean and variance of these distributions are given by

$$
E[\tau(i)]=E[\delta(i)]+E[k]
$$

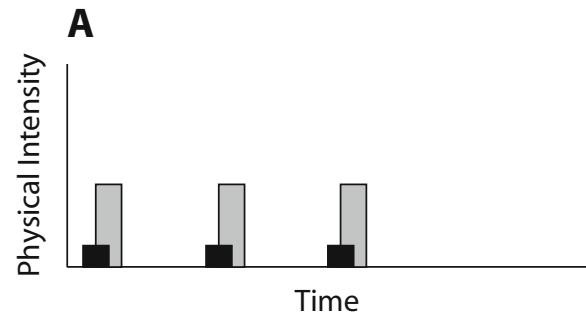

B

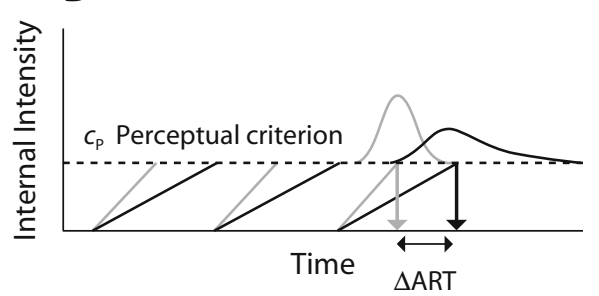

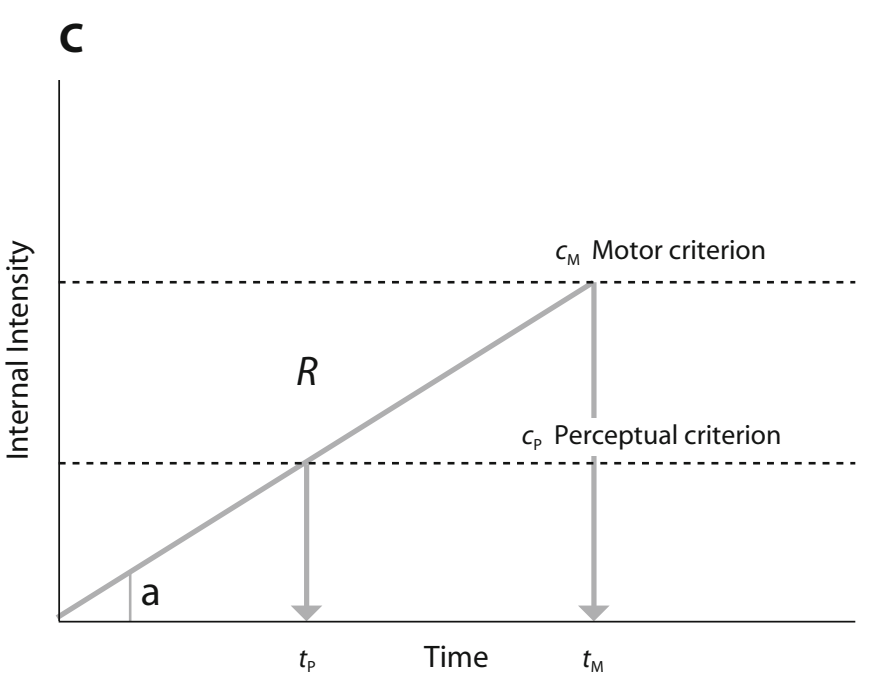

Figure 1. Rationale underlying the temporal anticipation task. (A) Temporal configuration of the sequence of high(gray bars) and low-saliency (black bars) stimulations. (B) The stimuli in panel A give rise to an internal activity whose buildup rate is proportional to their saliency: Internal responses to low-saliency stimuli (black oblique lines) reach a perceptual decision criterion $c_{\mathrm{P}}$ (dashed horizontal black line) later than do internal responses to high-saliency stimuli (gray oblique lines). Hence, the anticipatory response time (ART) for the low-saliency stimulus (black arrow) will be delayed by as much, with respect to the ART for the high-saliency (gray arrow) stimulus, with the ART difference ( $\triangle \mathrm{ART})$ proportional to the stimulus saliency difference. (C) The same internal response built up as in panel $B$, but with distinct perceptual and motor decision criteria ( $c_{\mathrm{P}}$ and $c_{\mathrm{M}}$, respectively; dashed lines). According to this scheme, a change in stimulus intensity (gray vs. black oblique lines in panel B) will yield a larger effect on the motor $(\Delta R T)$ than on the perceptual $(\triangle \mathrm{ART})$. See the text for more details. 


$$
\operatorname{Var}[\tau(i)]=\operatorname{Var}[\delta(i)]+\operatorname{Var}[k] .
$$

In the most general case, testing decision models of RT and/or ART requires a model of $\delta(i)$, but also of $k$. If we focus on the first and second moments of the RT distributions, the normalization procedure described below permits the canceling out of $k$. Furthermore, for a class of simple models such as the ones developed here, it is possible to decompose the decisional random variable (or its mean and variance) into the product of a criterion-only dependent function and an intensity-only dependent function. From this decomposed expression, it is easy to derive predictions of the relationship between ART and RT means and variances based only on the respective decision criteria. This procedure would not hold for more realistic models (positing a random starting point of the internal response and noisy decision criteria; Brown \& Heathcote, 2008) that require the explicit modeling of $\delta(i)$ and $k$.

The normalization procedure consists of subtracting the mean, $E[\tau(0)]$, and variance, $\operatorname{Var}[\tau(0)]$, of each observer's RT and ART response distributions for the highest contrast stimulus $(i=0)$ from his/her means and variances of the corresponding response distributions for the remaining contrasts - that is,

and

$$
{ }^{*} \mu_{\tau}(i)=E[\tau(i)]-E[\tau(0)]
$$

$$
{ }^{*} \sigma_{\tau}^{2}(i)=\operatorname{Var}[\tau(i)]-\operatorname{Var}[\tau(0)],
$$

with the asterisk standing for normalized and $i=\{1,2,3\}$ the index of the three remaining contrasts used in this study.

The predictions of the two models and the data are presented in terms of means and variances normalized via this procedure.

The simplest linear ballistic model. The simplest linear ballistic (SLB) model is similar to a number of existing models (Grice, 1972; Reddi et al., 2003; Reeves et al., 2005 ), in that it represents the accumulation of evidence within a trial as a deterministic linear function of time with a constant integration starting point (typically set to 0 ; for a critical review of such models, see Brown \& Heathcote, 2008). Accordingly, the internal response is $R=a(i) \times t$, with $t$ being the time and $a(i)$ the slope, a random variable (not necessarily Gaussian) dependent on stimulus intensity $i$. A decision to respond, $\delta$, is taken when the internal response $R$ exceeds the criterion $c$ - that is,

$$
\delta=\frac{c}{a(i)} .
$$

The RT distribution is thus given by

$$
\tau(i)=\frac{c}{a(i)}+k
$$

which has the following mean and variance:

and

$$
E[\tau(i)]=c \times E\left[\frac{1}{a(i)}\right]+E[k]
$$

$$
\operatorname{Var}[\tau(i)]=c^{2} \times \operatorname{Var}\left[\frac{1}{a(i)}\right]+\operatorname{Var}[k],
$$

where $E[k]$ and $\operatorname{Var}[k]$ cancel out after normalization. As is shown in Appendix A, SLB predicts a linear relationship between ${ }^{*} \mu_{\mathrm{RT}}(i)$ and ${ }^{*} \mu_{\mathrm{ART}}(i)$, as well as between ${ }^{*} \sigma_{\mathrm{RT}}^{2}(i)$ and ${ }^{*} \sigma_{\mathrm{ART}}^{2}(i)$, with slopes given by the ratio between the corresponding criteria and by the square of this ratio, respectively:

$$
{ }^{*} \mu_{\mathrm{ART}}(i)=\left(\frac{c_{\mathrm{ART}}}{c_{\mathrm{RT}}}\right) \times{ }^{*} \mu_{\mathrm{RT}}(i)
$$

and

$$
{ }^{*} \sigma_{\mathrm{ART}}^{2}(i)=\left(\frac{c_{\mathrm{ART}}}{c_{\mathrm{RT}}}\right)^{2} \times{ }^{*} \sigma_{\mathrm{RT}}^{2}(i) .
$$

Wald model. Unlike SLB, the Wald model represents the evidence accumulated over time as a stochastic process: At each time step (e.g., each millisecond), the observer is given some evidence, modeled as a normal distribution of mean $a(i)$ determined by $i$, the intensity of the stimulus. The decision to respond, $\delta$, is taken as the sum of these random variables exceeds a certain criterion $c$. For $a(i)<<c$, and as the time steps get increasingly small, the probability density function of the number of steps needed to exceed $c$ (i.e., $\delta$, the time needed to reach the decision) can be approximated by the Wald distribution (Heathcote, Brown, \& Cousineau, 2004; Wald, 1947):

$$
w(\delta)=\frac{c}{\sqrt{2 \pi \delta^{3}}} \times \exp \left\{-\frac{[c-a(i) \times \delta]^{2}}{2 \delta}\right\} .
$$

The mean and variance of the RT distribution under the Wald model are given by

$$
E[\tau(i)]=\frac{c}{a(i)}+E[k]
$$

and

$$
\operatorname{Var}[\tau(i)]=\frac{c}{a(i)^{3}}+\operatorname{Var}[k]
$$

The SLB and Wald models differ only in that the latter predicts the same slopes relating the normalized means (Equation 9) and the normalized variances - that is,

$$
{ }^{*} \sigma_{\mathrm{ART}}^{2}(i)=\left(\frac{c_{\mathrm{ART}}}{c_{\mathrm{RT}}}\right) \times{ }^{*} \sigma_{\mathrm{RT}}^{2}(i) .
$$

Modeling the ART task. The relationship between the ART and RT tasks predicted by either of the two models depends critically on how observers perform the ART task. When the interstimulus interval is constant throughout an experimental block and observers are told so (as in the present experiments; see the Method section), one strategy would be that once this interval is internalized (within a few trials), observers reproduce it thereafter without reassessing it on each new trial. In this case, ART and RT tasks become virtually identical, since they measure an RT relative to a unique start stimulus - that is, the second stimulus in the ART and the third in the RT stimulation sequences (see the Method section). This response strategy will be referred to as a simple detection strategy (SDS). The predictions presented above were derived assuming this SDS. 
Alternatively, subjects may evaluate the temporal interval between the first two stimulations on each trial and attempt to reproduce it by starting with their detection of the second stimulus in the sequence of three. This behavior is referred to hereafter as a double-detection strategy (DDS). It can be shown (see Appendix B) that relative to the predictions under the first strategy (SDS), the mean of ART is unaffected under DDS but that the ART variance is increased by a factor of five. In this case, the slope of the derived linear relationship between ART and RT variances (but not means) is also five times steeper for both SLB and Wald.

Be that as it may, SLB and Wald models predict linear, zero-intercept relationships both between the normalized ART and RT means and between the corresponding variances. The two models predict the same slope for the relationship between the normalized ART-RT means (see Equation 9)-that is,

$$
\frac{c_{\mathrm{ART}}}{c_{\mathrm{RT}}},
$$

but different slopes between the corresponding normalized variances (see Equations 10 and 14). The slope of the fitted zero-intercept line on the normalized means will thus be a test of the hypothesis of distinct task-specific (i.e., perceptual vs. motor) decision criteria, whereas the ratio between this slope and the slope on the normalized variances will, possibly, discriminate between the SLB and Wald models.

\section{METHOD}

\section{Stimuli}

The stimuli were 4 cycles/deg vertical Gabor patches with a standard deviation of $4^{\circ}$ presented on a virtual $6^{\circ}$-radius circle around a white fixation cross at a mean luminance of $40 \mathrm{~cd} / \mathrm{m}^{2}$. The contrast of the Gabor carrier was either .1, .2, .4, or .8, and it was fixed throughout an experimental block. The Gabors were displayed on a 19-in. CRT monitor $(1,024 \times 768$ pixels $)$ with a $100-\mathrm{Hz}$ refresh rate located $60 \mathrm{~cm}$ from the observer. In the ART experiment, the six Gabors were presented by successive pairs that remained on the screen, with the Gabors of each pair located at the opposite vertices of a hexagon. The presentation order was kept constant throughout the experiment, with the Gabor pair on the vertical meridian always the last (Steps 1-3 in Figure 2A). In the RT experiment, the first two pairs were presented simultaneously, followed at a random interval by the third pair. Stimulus presentation and response recording were controlled using the Psychophysics Toolbox (Brainard, 1997; Pelli, 1997) under MATLAB R14.

\section{Procedure}

In the ART task, the stimulus onset asynchrony (SOA) between the first and second pairs $\left(\mathrm{SOA}_{1-2}\right)$ equaled the SOA between the second and third pairs $\left(\mathrm{SOA}_{2-3}\right)$ and was kept constant at $500 \mathrm{msec}$. The observers were instructed to press a key simultaneously with the presentation of the third pair that completed the hexagonal display of the Gabor patches (Mamassian, 2006, 2008). A block of 20 training trials was performed by each subject to ensure that he or she properly understood the task.

In the RT task, the first two pairs were presented simultaneously $\left(\mathrm{SOA}_{1-2}=0\right)$, and the observers were required to perform a speeded keypress to the appearance of the third pair, whose onset was unpredictable. To counteract the influence of the foreperiod on RT (Luce,

\section{A}

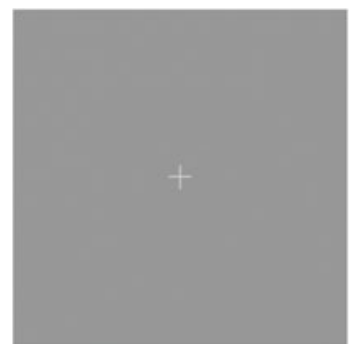

Foreperiod

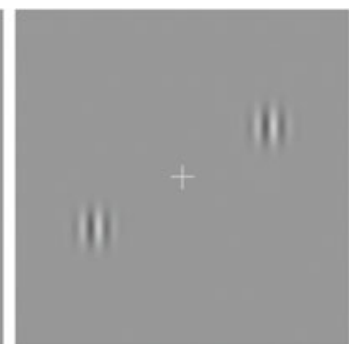

Step 1

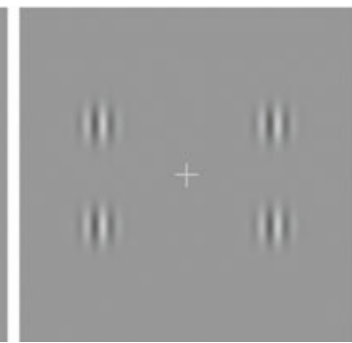

Step 2

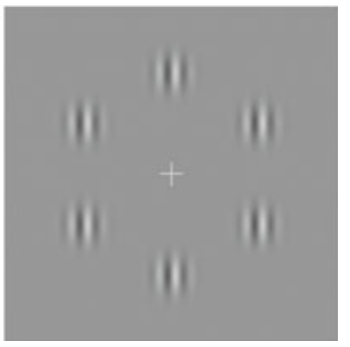

Step 3

B
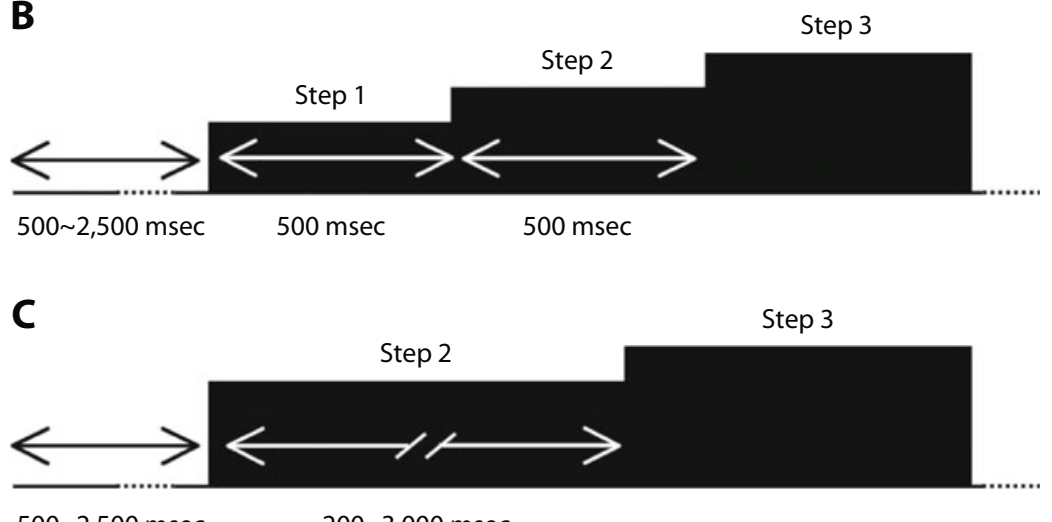

$500 \sim 2,500 \mathrm{msec}$

$200 \sim 3,000$ msec

Figure 2. One-trial sequence illustrating the spatial configuration of the stimuli (A) and their temporal organization in the anticipation $(B)$ and response time $(C)$ tasks. 
1986), the probability density function for the random $\mathrm{SOA}_{2-3}$ was distributed as a decaying exponential with a mean of $500 \mathrm{msec}$, truncated to a maximum of 3,000 msec plus a constant 200 -msec foreperiod.

In both tasks, the observers initiated each trial by pressing a key. The duration between this press and the onset of either the first pair (ART) or the first two pairs (RT) varied randomly according to an exponential distribution with a mean of $500 \mathrm{msec}$, truncated to a maximum of 2,000 msec plus a constant 500 -msec foreperiod.

One experimental block consisted of 50 trials and was specified in terms of the task (ART vs. RT) and the contrast of the Gabors $(.1, .2, .4$, and .8$)$; this yielded eight different experimental conditions. The nature of the task (ART vs. RT) was indicated on the screen at the beginning of each block. Each of the eight experimental blocks was run twice, and their sequence was randomized for each observer. Hence, each experimental point (mean RT, mean ART, and their associated variances) was computed out of 100 trials. The observers could take breaks between each block (every $3 \mathrm{~min}$ ), but not within a block (unless they did not press any key). The complete experiment lasted about $45 \mathrm{~min}$.

\section{Observers}

Eight right-handed observers ( 3 women, 5 men, including the first and last author; 24-54 years old) participated in the present experiments.

\section{RESULTS}

\section{Comparison of ART-RT Data and Models}

The raw data consisted of 64 (ART and RT) RT distributions ( 8 subjects $\times 4$ contrasts $\times 2$ tasks) with about 100 datum points each; values two times larger than the interquartile range of each sample were excluded $(M \pm S E=$ $6.33 \% \pm 0.630 \%$ ). A bootstrapping procedure was used to assess the reliability of the means and variances of each of these 64 distributions: The mean and variance of 50 trials randomly drawn (with replacement) from each of these 64 distributions were computed, and the procedure was repeated $10^{5}$ times. This yielded, for each of the 64 measured distributions, a distribution of means and a distribution of variances ( $10^{5}$ values each), each with its own mean $\left(E[\mu]\right.$ and $\left.E\left[\sigma^{2}\right]\right)$ and standard deviation $(S D[\mu]$ and $\left.S D\left[\sigma^{2}\right]\right)$, the latter being a measure of the reliability of $E[\mu]$ and of $E\left[\sigma^{2}\right]$, respectively.

As was explained in the Modeling the RT-ART Relationship section (Equations 4 and 5), these means of means $\left(E\left[\mu_{i}\right]\right)$ and means of variances $\left(E\left[\sigma_{i}^{2}\right]\right)$ were normalized to cancel out any task- and observer-specific random variable. These normalized values will be referred to as ${ }^{*} \mu_{\mathrm{RT}}$ and ${ }^{*} \sigma_{\mathrm{RT}}^{2}$ for the RT data and ${ }^{*} \mu_{\mathrm{ART}}$ and ${ }^{*} \sigma_{\mathrm{ART}}^{2}$ for the ART data. The ${ }^{*} \mu_{\mathrm{ART}}$ and ${ }^{*} \sigma_{\mathrm{ART}}^{2}$ datum points for each observer are shown separately in Figures 3A and 3B (different symbols for the 8 observers) as a function of the corresponding ${ }^{*} \mu_{\mathrm{RT}}$ and ${ }^{*} \sigma_{\mathrm{RT}}^{2}$. Each plot contains 24 datum points (i.e., 8 observers $\times 3$ contrasts, with the fourth contrast sacrificed for the normalization). Heavy lines are linear, zerointercept fits, as required by both SLB and Wald models.

Since the datum points were noisy along both the $x$ and $y$ dimensions, the fit was done with a weighted total least squares algorithm (Krystek \& Anton, 2007, 2008), where the weights associated with each datum point were $S D[\mu]$ and $S D\left[\sigma^{2}\right]$ and are shown as horizontal and vertical error bars, respectively.

The slope of the line fitted to the ${ }^{*} \mu_{\mathrm{ART}}$ versus ${ }^{*} \mu_{\mathrm{RT}}$ data (Figure $3 \mathrm{~A}$ ) is .64, with a $95 \%$ confidence interval ranging from .48 to .80 ; the equivalent slope for the ${ }^{*} \sigma_{\mathrm{ART}}^{2}$ versus ${ }^{*} \sigma_{\mathrm{RT}}^{2}$ data (Figure 3B) is .59 , with a $95 \%$ confidence interval ranging from .26 to .92 . Consequently, both slopes are significantly above zero and below one, evidencing a correlation between ART-RT variables while pointing to the difference between the decision processes underlying ART and RT responses.

The SLB and Wald models - assuming the SDS for the ART task (see the Modeling the RT-ART Relationship
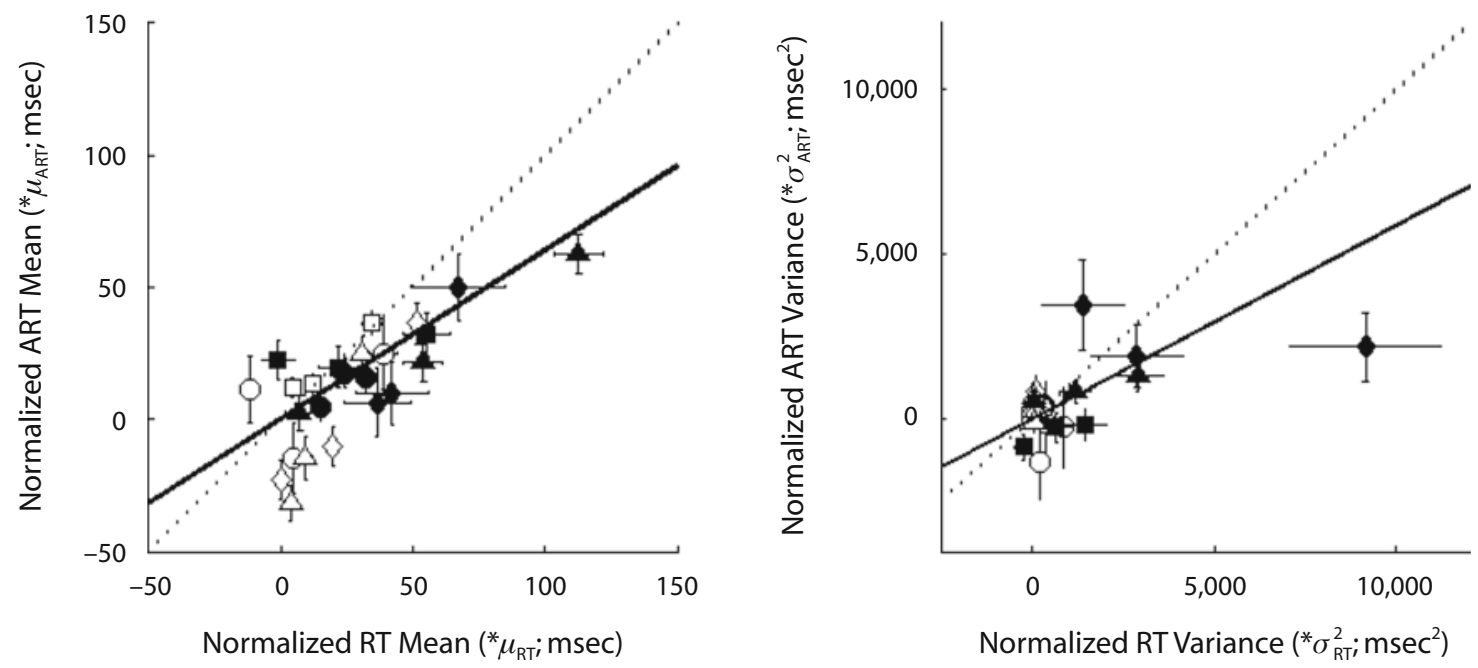

Figure 3. Normalized individual anticipatory response time (ART) means (" $\left.\mu_{\mathrm{ART}} ; \mathrm{A}\right)$ and variances (" $\sigma_{\mathrm{ART}}^{2}$; B) as a function of the corresponding normalized RT means $\left({ }^{*} \mu_{\mathrm{RT}}\right)$ and variances $\left({ }^{*} \sigma_{\mathrm{RT}}^{2}\right)$. Each datum point represents the bootstrapped data of 1 observer for one out of three contrast levels $(8 \times 3$ datum points), with the response to the highest contrast having been used as the reference. Straight solid lines are weighted total least squares linear fits performed on the complete data set. The weights are the standard deviations of each datum point determined via bootstrapping. Dotted lines are slope 1 diagonals. 
section)-differ in that the former predicts that the slope of the normalized variances is the square of the slope of the normalized means (Equations 9 and 10), whereas the latter predicts the same slope for the normalized means and variances (Equations 9 and 14). The present data and their linear fits (given their confidence intervals) are in good agreement with both models, so that they cannot discriminate between them. Critically, given either of the two models, the two independently estimated slopes indicate that the motor decision criterion is about 1.5 times higher than the perceptual decision criterion (precisely 1.30 and 1.70 as derived from the normalized variances under SLB and Wald models, respectively, and 1.56 as derived from the normalized means under either model).

The present data and their analysis point to the strong correlation between perceptual and motor latencies and to the fact that the means (as has frequently been reported) and variances of perceptual latencies are less affected by stimulus intensity than are the respective moments of the motor latencies. According to our analysis, the latter point is the consequence of the motor decision criterion being about 1.5 times higher than the perceptual one. It also supports the notion that observers' duration estimates in the ART task are internalized (within one experimental block) once and for all, rather than varying from trial to trial. In the latter case, the ${ }^{*} \sigma_{\mathrm{ART}}^{2}{ }^{*} \sigma_{\mathrm{RT}}^{2}$ ratio should have been 5 times higher than the one actually observed (cf. the Modeling the ART Task subsection and Appendix B).

To wrap it up, the present results and their analysis are in accord with a general model positing that perceptual and motor decisions are based on the same internal signal but are made with respect to distinct criteria, with the motor criterion 1.5 times higher than the perceptual criterion (Cardoso-Leite et al., 2007; Ejima \& Ohtani, 1987; Miller \& Schwarz, 2006; Sanford, 1974).

\section{Comparing TOJ and ART Paradigms}

On the basis of the observations above and as was mentioned in the introduction, the present ART task presents a number of advantages over the more traditional TOJ task: It is based on the use by observers of one single unambiguous temporal cue, is void of putative low-level interactions between the two stimulations required for TOJ, and is time constrained just as the RT but not as the TOJ task. Consequently, estimates of the decision moments derived from ART should be more readily comparable to those derived from RT. A direct comparison between the reliabilities of the perceptual-motor relationship as assessed using ART and TOJ tasks is hindered by the fact that they involve different stimulations. To make such a direct (although qualitative) comparison possible, ART data $\left(\mu_{\mathrm{ART}}\right.$ and $\left.\sigma_{\mathrm{ART}}\right)$ were converted into a TOJ-like format. To this end, we computed the difference in mean ART $\left[\Delta \mu_{\mathrm{ART}}(i, j)\right]$ between all possible binary $(i, j)$ combinations of stimulus intensities $\left[\mu_{\mathrm{ART}}(i)-\mu_{\mathrm{ART}}(j)\right]$. They are displayed in Figure $4 \mathrm{~A}$ as a function of the corresponding differences in mean RT $\left[\Delta \mu_{\mathrm{RT}}(i, j)=\mu_{\mathrm{RT}}(i)-\mu_{\mathrm{RT}}(j)\right]$. This format is equivalent to the PSS versus $\Delta \mu_{\mathrm{RT}}(i, j)$ plot in Figure $4 \mathrm{~A}^{\prime}$ from Cardoso-Leite et al. (2007). Similarly, Figure 4B displays the square roots of the sums of variances for all pos- sible stimulus intensity binary combinations $\left[\hat{\sigma}_{\mathrm{ART}}(i, j)=\right.$ $\left.\sqrt{\sigma_{\mathrm{ART}}^{2}(i)+\sigma_{\mathrm{ART}}^{2}(j)}\right]$ against the corresponding calculations for $\sigma_{\mathrm{RT}}$. Under this format, the present standard deviations are theoretically equivalent to those assessed in Cardoso-Leite et al.'s TOJ study presented in Figure 4B' ${ }^{\prime}{ }^{7}$

The converted ART data are clearly less scattered than the TOJ data (compare the 95\% confidence intervals in Figures $4 \mathrm{~A}$ and $4 \mathrm{~B}$ with those in Figures $4 \mathrm{~A}^{\prime}$ and $4 \mathrm{~B}^{\prime}$, dashed curves). Note that the slopes of the linear regressions ${ }^{8}$ through the $\Delta \mu_{\mathrm{ART}}(i, j)$ versus $\Delta \mu_{\mathrm{RT}}(i, j)$ (Figure 4A; slope, $0.631)$ and the $\hat{\sigma}_{\mathrm{ART}}(i, j)$ versus $\hat{\sigma}_{\mathrm{RT}}(i, j)$ (Figure 4B; slope, $0.437)$ data are below $1\left(p_{\mu}<.001 ; p_{\sigma}<.001\right)$ and comparable. This is not the case for the regressions through the PSS versus $\Delta \mu_{\mathrm{RT}}(i, j)$ (Figure $4 \mathrm{~A}^{\prime}$; slope, 0.475 ) and the $\sigma_{\mathrm{TOJ}}(i, j)$ versus $\hat{\sigma}_{\mathrm{RT}}(i, j)$ (Figure 4B'; slope, 1.466) data. It is notable that this gain in consistency is achieved at a lesser cost, since the assessment of a TOJ psychometric function is substantially more time consuming than the assessment of ART.

\section{DISCUSSION}

Perceptual and motor latencies have most often been measured and compared under unmatched stimulation conditions. Typically, differences in RT to one stimulus at a time have been compared with PSS inferred from TOJs of (by necessity) two successive stimuli. Masking, apparent motion, and/or attentional shift effects may interfere with the TOJ task, but not with the RT task, and should account at least in part for the observed discrepancies between the observed perceptual and motor performances. The present ART-RT paradigm minimizes the stimulation and response differences between the two tasks, thereby avoiding some uncontrolled sources of noise. In terms of data dispersion, a qualitative comparison between the results yielded by the present ART paradigm and the TOJ paradigm used by Cardoso-Leite et al. (2007) is indeed to the advantage of the former (Figure 4). This is not to say that ART is the "ultimate" perceptual latency measurement technique. In addition to using a stimulation sequence not entirely matched to the one used in the RT task, ART and RT measurements cannot be paired on a trial-by-trial basis, a procedure taken advantage of by Cardoso-Leite et al. Also, in the general case, the derivation of perceptual latencies from ART requires a model of time perception (bypassed here by the data normalization procedure; see the Modeling the RT-ART Relationship section).

Linear fits on the normalized means and variances of ART against the corresponding RT statistics yield slopes of .64 and .59, respectively. The .64 slope of the linear fit of the ART versus RT normalized means is in line with results in the literature showing that intensity (or contrast) manipulations affect the mean of perceptual latencies about 1.5 times less than they affect the mean of motor latencies (e.g., Jaśkowski, 1992; Jaśkowski \& Verleger, 2000; Roufs, 1974). The .59 slope of the linear relationship between ART and RT normalized variances is consistent with this differential effect of stimulus intensity on perceptual and motor latencies. This was not the case in Cardoso-Leite et al.'s (2007) study, in which the variance of the perceptual moments derived from TOJ was affected 
A

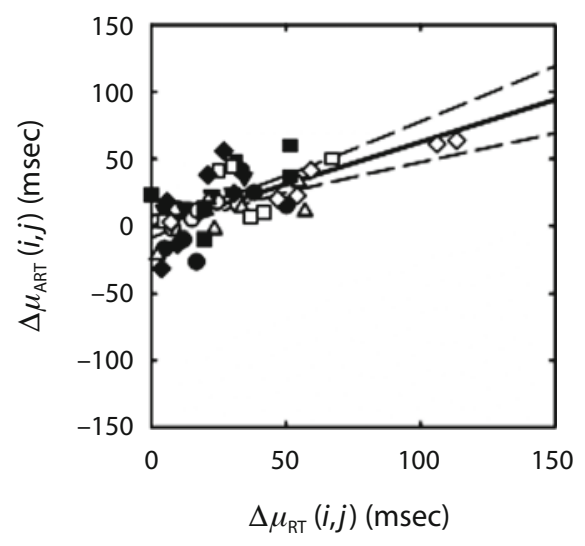

$A^{\prime}$

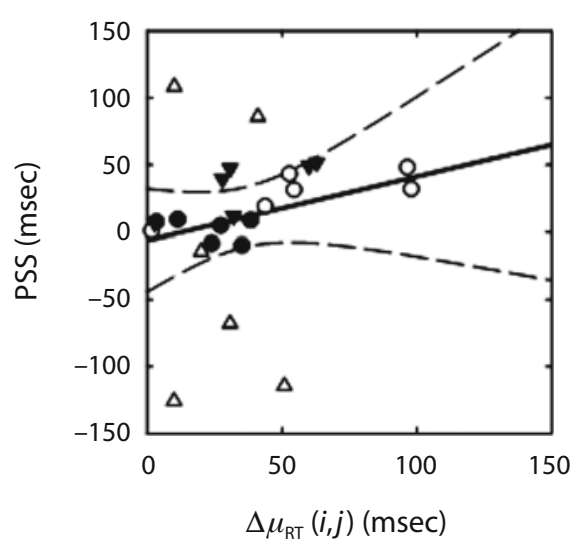

B

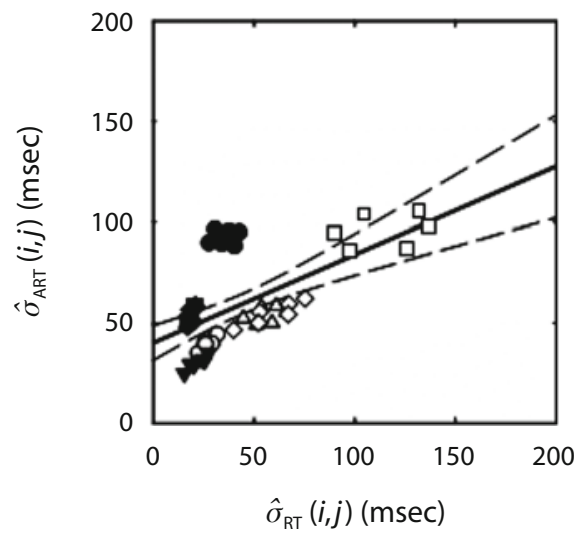

$\mathbf{B}^{\prime}$

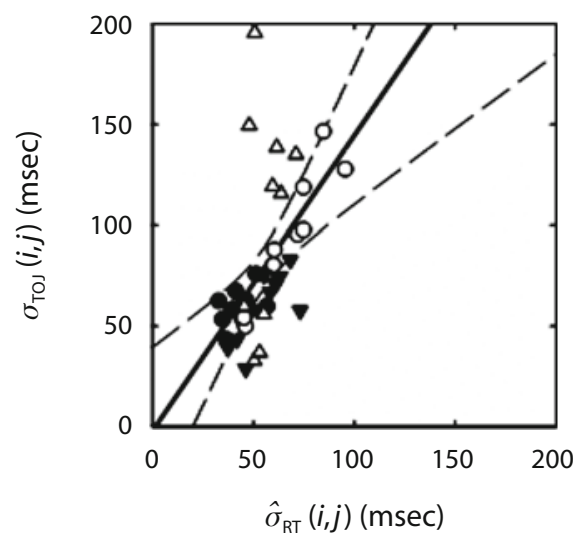

Figure 4. Relative mean perceptual versus motor times $\left(A, A^{\prime}\right)$ and their associated standard deviations $\left(B, B^{\prime}\right)$ as derived from the present data $(A, B$; see the text) and as measured in a temporal order judgment-response time (TOJ-RT) study by Cardoso-Leite, Gorea, and Mamassian (2007; $\left.\mathbf{A}^{\prime}, \mathbf{B}^{\prime}\right)$. Different symbols are for different observers. Straight solid lines are linear regressions. Dashed curves show $95 \%$ confidence intervals. PSS, point of subjective simultaneity.

more by stimulus intensity than was the variance of the motor latencies derived from RT. The incongruence could reflect the imperfect match between the RT and TOJ tasks. This possibility is reinforced by the fact that, given the observed relationship between the mean latencies derived from RT and TOJ, the relationship of the corresponding variances is incongruent with the one predicted by either of the SLB and Wald models tested in this study.

The slope of the relationship between ART and RT variances as predicted by either of these two models depends critically on observers' response strategy in the ART task. Indeed, observers may use an internalized reference duration throughout an experimental ART block or may compute this reference on a trial-by-trial basis. In the latter case, the two models predict that the slope of the relationship between ART and RT variances should be five times larger than the slope of the relationship between ART and RT means (see Appendix B). Since this is clearly rejected by the data, one should conclude in favor of the first alternative. If so, the present ART task can be looked upon as virtually identical to the RT task, since it involves a response to just a single stimulus.
The present data do not differentiate between the SLB and Wald models. These models differ in that they predict that the slope of the relationship between ART-RT variances is equal to (Wald) or is the square of (SLB) the slope of the relationship between ART-RT means. The confidence intervals of the linear fits of the observed means and variances are too large to reject either model. On the other hand, these confidence intervals are such that the observed slopes are statistically larger than 0 and smaller than 1 (.64 and .59). The difference from 0 is evidence of a significant correlation between perceptual (ART) and motor (RT) responses. The difference from 1 implies that perceptual and motor latencies as measured with ART and RT are not identical. The specific values of these slopes are compatible with the predictions of both SLB and Wald decision models. These models share the same backbone assumptionsnamely, that both perceptual and motor decisions are taken on evidence from a unique integration-to-bound process but that this evidence level differs between the two decisions. In particular, the larger effects of stimulus saliency on both the mean and variance of RT, as compared with the effects on the mean and variance of ART, respectively, indicate 
that motor decisions are taken at a higher evidence level (i.e., decision criterion) - hence, after a longer delay - than are perceptual decisions. The two independently estimated slopes (for the means and variances) consistently indicate that the motor decision criterion is about 1.5 times higher than the perceptual decision criterion (in fact, 1.30 and 1.70 as derived from the normalized variances under SLB and Wald models, respectively, and 1.56 as derived from the normalized means under either model).

Motor response criteria higher than perceptual criteria have been suggested by a number of authors (CardosoLeite et al., 2007; Ejima \& Ohtani, 1987; Miller \& Schwarz, 2006; Sanford, 1974). Miller and Schwarz argued that higher motor than perceptual criteria are recommended for perceptual-motor optimality (i.e., the best balance between maximum correct perceptual response rate and minimum false alarm rate for the motor responses). The general idea underlying such a claim can be understood in reference to action and perception having different loss functions (Maloney, 2002). It goes along with the no less general claim that, within a relevant temporal window, the quality of information accrues over time and that different response systems may access it at different instants (Hunt, von Mühlenen, \& Kingstone, 2007; Posner, 1978).

The motor and perceptual decision levels need not, however, be "hardwired" decision variables, so that their relative values may depend on both stimulation and contextual effects. Waszak et al. (2007) measured RT to a composite stimulation in which the first of two sequential stimuli (S1) was at threshold and was presented on only $50 \%$ of the trials, whereas the second stimulus (S2) had a constant, highly supraliminar contrast and was always present. Subsequent to their RT response, subjects had to indicate whether S1 was present or not, so that motor and perceptual responses were matched on a trial-by-trial basis. In addition, S2 was spatially close to or far away from $\mathrm{S} 1$, so that it masked (metacontrast) or did not mask, respectively, the latter. Waszak et al. showed that the RTs associated with perceptual hits dropped with the intensity of S1 whether S1 was or was not masked. Instead, the RTs associated with perceptual misses were independent of the intensity of S1 in the nonmasked condition (see also Waszak \& Gorea, 2004) but did drop with S1 intensity in the masked condition. These authors accounted for their results by posing that the perceptual criterion was below the motor criterion in the nonmasked condition and above it in the masked condition. Contextual factors meant to modulate perceptual and/ or motor criteria (e.g., signal probability or cost function) have been manipulated in a number of studies, although, with one exception (Waszak \& Gorea, 2004), not jointly assessed (e.g., Carpenter \& Williams, 1995; Green \& Swets, 1966; Janssen \& Shadlen, 2005; Mamassian, 2006, 2008; Trommershäuser, Landy, \& Maloney, 2006).

As a final note, it is worth mentioning that the distinction between perception and action remains debatable from both a methodological and a conceptual standpoint. By definition, detection (or discrimination) tasks measure perceptual performances (assessed with yes/no or forced choice paradigms) typically assessed under unconstrained RT conditions. As the response is progres- sively speeded (and "perceptual" performance drops), the task is regarded as being more of the "motor" type. The perceptual-motor classification is therefore volatile. Speeded or not, a behavioral response is ultimately motor and necessarily (at least in the laboratory) triggered by a sensory stimulation. Clarifying the ambiguity of the perception-action distinction and of its twin conscious (vision for perception) versus unconscious (vision for action) dichotomy was not the purpose of the present study. Instead, its achievement is to show that performances in two tasks presumably isolating perceptual and motor aspects of one's behavior are strongly correlated. In fact, since visual perception is inherently ambiguous (Helmholtz, 1867), how can it be that perception and action typically reach identical conclusions if they rely on independent processes (Franz et al., 2000)?

\section{Conclusion}

The present study introduced an alternative technique for assessing perceptual latencies (ART) in conjunction with motor latencies and showed that it is more reliable, less subject to uncontrolled, interfering low-level factors, and more efficient than the standard TOJ technique. Its use in comparing perceptual and motor latencies demonstrated that these behaviors are highly correlated and can be accurately described by either of two models positing a shared internal signal and two distinct decision criteria, with the motor criterion higher than the perceptual one. The relationship between these distinct decision variables may, nonetheless, be modifiable by stimulation conditions and/or contextual effects.

\section{AUTHOR NOTE}

This work was supported by Grants ANR-06-NEURO-042-01 and BQR Paris Descartes Univ. 2006 to A.G. We thank Simon Barthelmé, Joshua Solomon, our action editor, and the two anonymous reviewers for their helpful contributions. Correspondence concerning this article should be addressed to P. Cardoso-Leite, Laboratoire Psychologie de la Perception, Université Paris Descartes and CNRS, 45 rue des SaintsPères, 75006 Paris, France (e-mail: pdrcardoso@gmail.com).

\section{REFERENCES}

Allan, L. G. (1998). The influence of the scalar timing model on human timing research. Behavioural Processes, 44, 101-117.

AschersLeben, G. (2002). Temporal control of movements in sensorimotor synchronization. Brain \& Cognition, 48, 66-79.

Aschersleben, G., \& Müsseler, J. (1999). Dissociations in the timing of stationary and moving stimuli. Journal of Experimental Psychology: Human Perception \& Performance, 25, 1709-1720.

BAMBER, D. (1979). State-trace analysis: A method of testing simple theories of causation. Journal of Mathematical Psychology, 19, 137-181.

Boring, E. G. (1942). Sensation and perception in the history of experimental psychology. New York: Appleton-Century-Crofts.

Brainard, D. H. (1997). The Psychophysics Toolbox. Spatial Vision, 10, 433-436.

Brown, S. D., \& Heathcote, A. (2008). The simplest complete model of choice response time: Linear ballistic accumulation. Cognitive Psychology, 57, 153-178.

Buonomano, D. V. (2000). Decoding temporal information: A model based on short-term synaptic plasticity. Journal of Neuroscience, $\mathbf{2 0}$, 1129-1141.

Cardoso-Leite, P., Gorea, A., \& Mamassian, P. (2007). Temporal order judgment and simple reaction times: Evidence for a common processing system. Journal of Vision, 7, 1-14. 
Carpenter, R. H. S., \& Williams, M. L. L. (1995). Neural computation of log likelihood in control of saccadic eye movements. Nature, 377, 59-62.

Doenring, D. G. (1961). Accuracy and consistency of time-estimation by four methods of reproduction. American Journal of Psychology, 74, 27-35.

EJima, Y., \& OHTANi, Y. (1987). Simple reaction time to sinusoidal grating and perceptual integration time: Contributions of perceptual and response processes. Vision Research, 27, 269-276.

Franz, V. H., Gegenfurtner, K. R., Bülthoff, H. H., \& Fahle, M. (2000). Grasping visual illusions: No evidence for a dissociation between perception and action. Psychological Science, 11, 20-25.

Gegenfurtner, K. R., \& Franz, V. H. (2007). A comparison of localization judgments and pointing precision. Journal of Vision, 7, 1-12.

Gibbon, J., \& Rutschmann, R. (1969). Temporal order judgment and reaction time. Science, $\mathbf{1 6 5}, 413-415$.

GLimcher, P. W. (2003). Decisions, uncertainty, and the brain: The science of neuroeconomics. Cambridge, MA: MIT Press.

Gold, J. I., \& Shadlen, M. N. (2001). Neural computations that underlie decisions about sensory stimuli. Trends in Cognitive Sciences, 5, 10-16.

Goodale, M. A., \& Milner, A. D. (1992). Separate visual pathways for perception and action. Trends in Neurosciences, 15, 20-25.

Green, D. M., \& Swets, J. A. (1966). Signal detection theory and psychophysics. New York: Wiley.

GRICE, G. R. (1972). Application of a variable criterion model to auditory reaction time as a function of the type of catch trial. Perception \& Psychophysics, 12, 103-107.

Heathcote, A., Brown, S., \& Cousineau, D. (2004). QMPE: Estimating Lognormal, Wald, and Weibull RT distributions with a parameterdependent lower bound. Behavior Research Methods, Instruments, \& Computers, 36, 277-290.

Helmholtz, H. von (1867). Handbuch der physiologischen Optik. Leipzig: Voss.

Hunt, A. R., von Mühlenen, A., \& Kingstone, A. (2007). The time course of attentional and oculomotor capture reveals a common cause. Journal of Experimental Psychology: Human Perception \& Performance, 33, 271-284.

JANSSEN, P., \& SHADLEN, M. N. (2005). A representation of the hazard rate of elapsed time in macaque area LIP. Nature Neuroscience, 8, 234-241.

JAŚKOWSKI, P. (1992). Temporal-order judgment and reaction time for short and long stimuli. Psychological Research, 54, 141-145.

JAŚKOWSKI, P. (1993). Temporal-order judgment and reaction time to stimuli of different rise times. Perception, 22, 963-970.

JAŚKOWSKI, P. (1996). Simple reaction time and perception of temporal order: Dissociations and hypotheses. Perceptual \& Motor Skills, 82, 707-730.

JAŚKOWSKI, P. (1999). Reaction time and temporal order judgment as measures of perceptual latency: The problem of dissociations. In G. Aschersleben, T. Bachmann, \& J. Müsseler (Eds.), Cognitive contributions to the perception of spatial and temporal events (pp. 265282). Amsterdam: Elsevier.

JAŚKOWSKI, P., \& VerLeger, R. (2000). Attentional bias toward lowintensity stimuli: An explanation for the intensity dissociation between reaction time and temporal order judgment? Consciousness \& Cognition, 9, 435-456.

JEANNEROD, M. (1997). The cognitive neuroscience of action. Oxford: Blackwell.

KANAI, R., \& Kamitani, Y. (2003). Time-locked perceptual fading induced by visual transients. Journal of Cognitive Neuroscience, 15, 664-672.

Karmarkar, U. R., \& Buonomano, D. V. (2007). Timing in the absence of clocks: Encoding time in neural network states. Neuron, 53, 427-438.

Klotz, W., \& Neumann, O. (1999). Motor activation without conscious discrimination in metacontrast masking. Journal of Experimental Psychology: Human Perception \& Performance, 25, 976-992.

Krystek, M., \& ANTON, M. (2007). A weighted total least-squares algorithm for fitting a straight line. Measurement Science \& Technology, 18, 3438-3442.

Krystek, M., \& Anton, M. (2008). A weighted total least-squares algorithm for fitting a straight line. Measurement Science \& Technology, 19, 079801

LENNIE, P. (1981). The physiological basis of variations in visual latency. Vision Research, 21, 815-824.
LUCE, R. D. (1986). Response times: Their role in inferring elementary mental organization. Oxford: Oxford University Press.

MaLoney, L. T. (2002). Statistical decision theory and biological vision. In D. Heyer \& R. Mausfeld (Eds.), Perception and the physical world: Psychological and philosophical issues in perception (pp. 145-189). New York: Wiley.

Mamassian, P. (2006). Visuo-motor synchrony [Abstract]. Journal of Vision, 6, 395

Mamassian, P. (2008). Overconfidence in an objective anticipatory motor task. Psychological Science, 19, 601-606.

Mauk, M. D., \& Buonomano, D. V. (2004). The neural basis of temporal processing. Annual Review of Neuroscience, 27, 307-340.

Merigan, W. H., \& Maunsell, J. H. (1993). How parallel are the primate visual pathways? Annual Review of Neuroscience, 16, 369-402.

Miller, J., \& Schwarz, W. (2006). Dissociations between reaction times and temporal order judgments: A diffusion model approach. Journal of Experimental Psychology: Human Perception \& Performance, 32, 394-412.

Milner, A. D., \& Goodale, M. A. (1995). The visual brain in action. Oxford: Oxford University Press.

Miyazaki, M., Yamamoto, S., Uchida, S., \& Kitazawa, S. (2006) Bayesian calibration of simultaneity in tactile temporal order judgment. Nature Neuroscience, 9, 875-877.

Neumann, O., Esselmann, U., \& Klotz, W. (1993). Differential effects of visual-spatial attention on response latency and temporalorder judgment. Psychological Research, 56, 26-34.

Pelli, D. G. (1997). The VideoToolbox software for visual psychophysics: Transforming numbers into movies. Spatial Vision, 10, 437-442.

Pisella, L., Binkofski, F., Lasek, K., Toni, I., \& Rossetti, Y. (2006). No double-dissociation between optic ataxia and visual agnosia: Multiple sub-streams for multiple visuo-manual integrations. Neuropsychologia, 44, 2734-2748.

PosNer, M. I. (1978). Chronometric explorations of mind. Hillsdale, NJ: Erlbaum.

Prinz, W. (1992). Why don't we perceive our brain states? European Journal of Cognitive Psychology, 4, 1-20.

Reddi, B. A. J., Asrress, K. N., \& Carpenter, R. H. S. (2003). Accuracy, information, and response time in a saccadic decision task. Journal of Neurophysiology, 90, 3538-3546.

Reeves, A., Santhi, N., \& DeCaro, S. (2005). A random-ray model for speed and accuracy in perceptual experiments. Spatial Vision, 18, 73-83.

Rossetti, Y., Pisella, L., \& Vighetto, A. (2003). Optic ataxia revisited: Visually guided action versus immediate visuomotor control. Experimental Brain Research, 153, 171-179.

Roufs, J. A. J. (1963). Perception lag as a function of stimulus luminance. Vision Research, 3, 81-91.

Roufs, J. A. J. (1974). Dynamic properties of vision: V. Perception lag and reaction time in relation to flicker and flash thresholds. Vision Research, 14, 853-869.

SANFORD, A. J. (1974). Attention bias and the relation of perception lag to simple reaction time. Journal of Experimental Psychology, 102, $443-446$.

SCHENK, T. (2006). An allocentric rather than perceptual deficit in patient D.F. Nature Neuroscience, 9, 1369-1370.

Schmidt, T., \& Vorberg, D. (2006). Criteria for unconscious cognition: Three types of dissociation. Perception \& Psychophysics, $\mathbf{6 8}$ 489-504.

Sternberg, S., \& Knoll, R. L. (1973). The perception of temporal order: Fundamental issues and a general model. In S. Kornblum (Ed.), Attention and performance IV (pp. 629-685). New York: Academic Press.

Stevens, L. T. (1886). On the time sense. Mind, 11, 393-404.

Stone, L. S., \& Krauzlis, R. J. (2003). Shared motion signals for human perceptual decisions and oculomotor actions. Journal of $\mathrm{Vi}$ sion, 3, 725-736.

TAPPE, T., NiePEL, M., \& Neumann, O. (1994). A dissociation between reaction time to sinusoidal gratings and temporal-order judgment. Perception, 23, 335-347.

Trommershäuser, J., Landy, M. S., \& Maloney, L. T. (2006). Humans rapidly estimate expected gain in movement planning. Psychological Science, 17, 981-988.

Vorberg, D., Mattler, U., Heinecke, A., Schmidt, T., \& Schwarz- 
BACH, J. (2003). Different time courses for visual perception and action priming. Proceedings of the National Academy of Sciences, 100, 6275-6280.

Wald, A. (1947). Sequential analysis. New York: Wiley.

Waszak, F., Cardoso-Leite, P., \& Gorea, A. (2007). Perceptual criterion and motor threshold: A signal detection analysis of the relationship between perception and action. Experimental Brain Research, 182, 179-188.

WASZAK, F., \& GoREA, A. (2004). A new look at the relationship between perceptual and motor responses. Visual Cognition, 11, 947-963.

Wearden, J. H. (2003). Applying the scalar timing model to human time psychology: Progress and challenges. In H. Helfrich (Ed.), Time and mind II: Information processing perspectives (pp. 21-39). Cambridge, MA: Hogrefe \& Huber.

\section{NOTES}

1. This operational classification leaves no room for the long-forgotten debate on the relationship between sensation and perception (see Boring, 1942).

2. This formalization, only alluded to in that study, corresponds to the simple ballistic model fully developed in the present study.

3. As will become clear below, this is not a critical issue, since our ART procedure is meant to measure relative perception latency, which is independent of the sign of the physical asynchrony between the tap and the stimulus to be synchronized with.

4. Inasmuch as observers comply with the synchronization task, visual perception latency (modulated by contrast) and proprioceptive perception latency (independent of visual contrast) may be such that for a given stimulus contrast, perceptual visuo-proprioceptive synchronicity may or may not match the physical synchronicity between stimulus and pressing onsets (e.g., Prinz, 1992).

5. As was noted by an anonymous reviewer, this type of analysis (expressing one dependent variable as a function of another) is known as state-trace analysis (Bamber, 1979).

6 . In the case of RT, $k$ may reflect, among other things, axonal conduction delays and the duration needed to perform the muscle contraction leading to the buttonpress. In the case of ART, $k$ also includes subjects' ability to estimate and reproduce a duration, a process modeled in many different ways (e.g., Allan, 1998; Buonomano, 2000; Karmarkar \& Buonomano, 2007; Mauk \& Buonomano, 2004; Wearden, 2003).

7. This reasoning is valid if, as in the TOJ-RT experiment, the nondecisional random variables affecting ART and RT are considered to be negligible.

8. Linear regressions on these data are inappropriate. We performed this analysis on the presently measured RT and ART means and standard deviations for the sake of this qualitative comparison with those (incorrectly) performed by Cardoso-Leite et al. (2007) on the RT versus TOJ data.

\section{APPENDIXA \\ Predictions of the SLB and Wald Models}

Assuming that observers use the SDS to perform the ART task (see Appendix B), the means and variances of both RT and ART response distributions, $\tau(i)$, under the SLB model are given by

and

$$
E[\tau(i)]=c \times E\left[\frac{1}{a(i)}\right]+E[k]
$$

$$
\operatorname{Var}[\tau(i)]=c^{2} \times \operatorname{Var}\left[\frac{1}{a(i)}\right]+\operatorname{Var}[k]
$$

and under the Wald model by

and

$$
E[\tau(i)]=c \times \frac{1}{a(i)}+E[k]
$$

$$
\operatorname{Var}[\tau(i)]=c \times \frac{1}{a(i)^{3}}+\operatorname{Var}[k] .
$$

Assuming the DDS for performing the ART task, the means and variances of the ART distribution are given by

and

$$
E[\tau(i)]=c \times E\left[\frac{1}{a(i)}\right]+E[k]
$$

$$
\operatorname{Var}[\tau(i)]=5 \times c^{2} \times \operatorname{Var}\left[\frac{1}{a(i)}\right]+\operatorname{Var}[k]
$$

under the SLB model and by

and

$$
E[\tau(i)]=c \times \frac{1}{a(i)}+E[k]
$$

$$
\operatorname{Var}[\tau(i)]=5 \times c \times \frac{1}{a(i)^{3}}+\operatorname{Var}[k]
$$

under the Wald model.

The normalized means and normalized variances are defined as

$$
{ }^{*} \mu_{\tau}(i)=E[\tau(i)]-E[\tau(0)]
$$

and

$$
{ }^{*} \sigma_{\tau}^{2}(i)=\operatorname{Var}[\tau(i)]-\operatorname{Var}[\tau(0)]
$$

where $\tau(0)$ refers to the RT distribution to the highest contrast stimulus. 


\section{APPENDIX A (Continued)}

Replacing $E[\tau(i)]$ and $\operatorname{Var}[\tau(i)]$ in Equations $\mathrm{A} 9$ and $\mathrm{A} 10$ with the formalization corresponding to the two decision models and for both ART strategies (SDS and DDS), the corresponding normalized means and variances are easily derived from Equations A1-A8. Under SLB and SDS, they are given by

$$
{ }^{*} \mu_{\tau}(i)=c \times\left(E\left[\frac{1}{a(i)}\right]-E\left[\frac{1}{a(0)}\right]\right)
$$

and

$$
{ }^{*} \sigma_{\tau}^{2}(i)=c^{2} \times\left(\operatorname{Var}\left[\frac{1}{a(i)}\right]-\operatorname{Var}\left[\frac{1}{a(0)}\right]\right) .
$$

Under Wald and SDS, they are given by

$$
{ }^{*} \mu_{\tau}(i)=c \times\left(\frac{1}{a(i)}-\frac{1}{a(0)}\right)
$$

and

$$
{ }^{*} \sigma_{\tau}^{2}(i)=c \times\left(\frac{1}{a(i)^{3}}-\frac{1}{a(0)^{3}}\right) .
$$

Under SLB and DDS,

$$
{ }^{*} \mu_{\tau}(i)=c \times\left(E\left[\frac{1}{a(i)}\right]-E\left[\frac{1}{a(0)}\right]\right)
$$

and

$$
{ }^{*} \sigma_{\tau}^{2}(i)=5 \times c^{2} \times\left(\operatorname{Var}\left[\frac{1}{a(i)}\right]-\operatorname{Var}\left[\frac{1}{a(0)}\right]\right) .
$$

And, finally, under Wald and DDS,

$$
{ }^{*} \mu_{\tau}(i)=c \times\left(\frac{1}{a(i)}-\frac{1}{a(0)}\right)
$$

and

$$
{ }^{*} \sigma_{\tau}^{2}(i)=5 \times c \times\left(\frac{1}{a(i)^{3}}-\frac{1}{a(0)^{3}}\right) .
$$

We now will present the derivation of Equations 9 and 10 in the main text; they express the normalized mean and variance of ART as a function of the normalized mean and variance of RT under SLB and given SDS. The derivation of the equivalent equations under Wald and SDS (Equations 9 and 14 in the main text), SLB and DDS (Equations B8-B9), and Wald and DDS (Equations B10-B11) is identical. The normalized mean and variance of RT are given by

$$
{ }^{*} \mu_{\mathrm{RT}}(i)=c_{\mathrm{RT}} \times\left(E\left[\frac{1}{a(i)}\right]-E\left[\frac{1}{a(0)}\right]\right)
$$

and

$$
{ }^{*} \sigma_{\mathrm{RT}}^{2}(i)=c_{\mathrm{RT}}^{2} \times\left(\operatorname{Var}\left[\frac{1}{a(i)}\right]-\operatorname{Var}\left[\frac{1}{a(0)}\right]\right),
$$

and the normalized mean and variance of ART are given by

and

$$
{ }^{*} \mu_{\mathrm{ART}}(i)=c_{\mathrm{ART}} \times\left(E\left[\frac{1}{a(i)}\right]-E\left[\frac{1}{a(0)}\right]\right)
$$

$$
{ }^{*} \sigma_{\mathrm{ART}}^{2}(i)=c_{\mathrm{ART}}^{2} \times\left(\operatorname{Var}\left[\frac{1}{a(i)}\right]-\operatorname{Var}\left[\frac{1}{a(0)}\right]\right),
$$

where $c_{\mathrm{RT}}$ and $c_{\mathrm{ART}}$ represent the RT and ART decision criteria, respectively. Rewriting the normalized mean and variance of RT and ART yields

$$
\left(E\left[\frac{1}{a(i)}\right]-E\left[\frac{1}{a(0)}\right]\right)=\frac{1}{c_{\mathrm{RT}}} \times{ }^{*} \mu_{\mathrm{RT}}(i)=\frac{1}{c_{\mathrm{ART}}} \times{ }^{*} \mu_{\mathrm{ART}}(i)
$$

and

$$
\left(\operatorname{Var}\left[\frac{1}{a(i)}\right]-\operatorname{Var}\left[\frac{1}{a(0)}\right]\right)=\frac{1}{c_{\mathrm{RT}}^{2}} \times{ }^{*} \sigma_{\mathrm{RT}}^{2}(i)=\frac{1}{c_{\mathrm{ART}}^{2}} \times{ }^{*} \sigma_{\mathrm{ART}}^{2}(i) .
$$




\section{APPENDIXA (Continued)}

It is easy to see that

$$
* \mu_{\mathrm{ART}}(i)=\left(\frac{c_{\mathrm{ART}}}{c_{\mathrm{RT}}}\right) \times * \mu_{\mathrm{RT}}(i)
$$

and

$$
* \sigma_{\mathrm{ART}}^{2}(i)=\left(\frac{c_{\mathrm{ART}}}{c_{\mathrm{RT}}}\right)^{2} \times{ }^{*} \sigma_{\mathrm{RT}}^{2}(i) .
$$

\section{APPENDIX B}

\section{Alternative ART Strategies}

The RT distribution $\tau(i)$ depends on, among other things, stimulus intensity $i$, the model of the decision process (i.e., SLB or Wald), and the response strategy used in the ART task. One ART response strategy, developed in the article, consists of reproducing an internalized duration at the moment of detection of the second stimulus in the sequence of three. This ART response strategy will be referred to as a simple detection strategy (SDS). Alternatively, subjects could evaluate on each trial the temporal interval between the first two stimulations and attempt to reproduce it, starting with their detection of the second one. Since this strategy implies the detection of the first and second stimulations, it will be referred to as a double-detection strategy (DDS). Here, we develop the predictions of the relationships between ART and RT means and variances under both SLB and Wald decision models based on the DDS.

Let $\delta_{1}, \delta_{2}$, and $\delta_{3}$ be the moments of detection of the first, second, and third stimuli (expressed relative to their respective physical onsets), and let $d$ be the physical interstimulus interval. The duration separating $\delta_{1}$ and $\delta_{2}$ is $\left(\delta_{2}+d\right)-\delta_{1}$, and the expected moment of detection of the third stimulus, $\delta_{3}$, is given by $\delta_{1}+2\left(\delta_{2}+d-\right.$ $\delta_{1}$ ). Rewriting this equation and inserting a random variable $k$ reflecting, among other things, a noisy duration estimation and reproduction process, the latency distribution of the keypresses in the ART task is given by

$$
\tau(i)=2 \delta_{2}(i)-\delta_{1}(i)+2 d+k,
$$

with its mean, expressed relative to the onset of the third stimulus, given by

$$
\begin{aligned}
& E[\tau(i)]=E\left[2 \delta_{2}(i)-\delta_{1}(i)+k\right] \\
& E[\tau(i)]=E\left[2 \delta_{2}(i)\right]-E\left[\delta_{1}(i)\right]+E[k] \\
& E[\tau(i)]=E[\delta(i)]+E[k]
\end{aligned}
$$

and its variance given by

$$
\begin{aligned}
\operatorname{Var}[\tau(i)] & =\operatorname{Var}\left[2 \delta_{2}(i)-\delta_{1}(i)+k\right] \\
\operatorname{Var}[\tau(i)] & =\operatorname{Var}\left[2 \delta_{2}(i)\right]+\operatorname{Var}\left[\delta_{1}(i)\right]+\operatorname{Var}[k] \\
\operatorname{Var}[\tau(i)] & =5 \times \operatorname{Var}[\delta(i)]+\operatorname{Var}[k] .
\end{aligned}
$$

Equations B1-B7 are independent of how $\delta$ is modeled.

Given the DDS for performing the ART task, the predicted relationships between the normalized mean and variance of the ART distribution and the normalized mean and variance of the RT distribution are given, under SLB, by

$$
{ }^{*} \mu_{\mathrm{ART}}(i)=\left(\frac{c_{\mathrm{ART}}}{c_{\mathrm{RT}}}\right) \times * \mu_{\mathrm{RT}}(i)
$$

and

$$
* \sigma_{\mathrm{ART}}^{2}(i)=5 \times\left(\frac{c_{\mathrm{ART}}}{c_{\mathrm{RT}}}\right)^{2} \times{ }^{*} \sigma_{\mathrm{RT}}^{2}(i)
$$

and, under Wald, by

$$
{ }^{*} \mu_{\mathrm{ART}}(i)=\left(\frac{c_{\mathrm{ART}}}{c_{\mathrm{RT}}}\right) \times{ }^{*} \mu_{\mathrm{RT}}(i)
$$

and

$$
{ }^{*} \sigma_{\mathrm{ART}}^{2}(i)=5 \times\left(\frac{c_{\mathrm{ART}}}{c_{\mathrm{RT}}}\right) \times{ }^{*} \sigma_{\mathrm{RT}}^{2}(i) .
$$

